\title{
Correlación entre el Puntaje Obtenido en la Prueba "Timed up and go" y Momentos Articulares del Miembro Inferior Registrados Durante la Transferencia de Sedente a Bípedo en Adultos Mayores con Antecedentes de Caídas Frecuentes
}

\author{
Correlation between the Scores of "Timed up and go" Test and Registered Joint Moments of \\ Lower Limb During the Sit-to-Stand Transfer in Elderly with Antecedents of Frequent Falls
}

"Guzmán, R. A.; " Salazar, H. A.; " Cea, A.; "**Melián, H. P.; *** Cordier, B. \& "Silvestre, R. A.

GUZMÁN, R. A.; SAlAZAR, H. A.; CEA, A.; MELIÁN, H. P.; CORDIER, B. \& SILVESTRE, R. A. Correlación entre el puntaje obtenido en la prueba "Timed up and go" y momentos articulares del miembro inferior registrados durante la transferencia de sedente a bípedo en adultos mayores con antecedentes de caídas frecuentes. Int. J. Morphol., 29(2):521-525, 2011.

RESUMEN: Los cambios producidos durante el envejecimiento predisponen al adulto mayor a las caídas frecuentes, en el ambiente clínico el riesgo de caída es valorado mediante pruebas clínicas que muchas veces carecen de poder analítico, por lo cual es necesario describir cual de dichas pruebas puede tener mayor relación con parámetros biomecánicos analíticos con la finalidad de conferirle a dichas pruebas funcionales tales características. El objetivo de este trabajo fue describir la existencia de correlación entre los puntajes obtenidos en la prueba funcional "Timed up and go" (TUG) y momentos articulares del miembro inferior obtenidos durante la ejecución de la transferencia de sedente a bípedo (TSB) en sujetos adultos mayores con antecedentes de caídas frecuentes. Se obtuvo una muestra de 30 voluntarios, todos adultos mayores con antecedentes de caídas frecuentes, los que fueron evaluados con la prueba funcional de TUG. También se evaluó mediante un sistema de análisis de movimiento la TSB donde se registraron los parámetros biomecánicos necesarios para determinar los momentos articulares del miembro inferior. Existió una correlación significativa $(r=-0,39$; $p=0,03)$ entre el puntaje obtenido en el TUG y el momento articular máximo de rodilla. Para la muestra evaluada, el tiempo de ejecución de la prueba "Timed up and go" fue indicador de la capacidad de generar momento articular por parte de los músculos flexo-extensores de rodilla en sujetos con antecedentes de caídas frecuentes.

PALABRAS CLAVE: Caídas; Ancianos; Momento; Biomecánica.

\section{INTRODUCCIÓN}

El proceso de envejecimiento de la población se caracteriza por el aumento de la población mayor de 60 años (INE, 2007). En la medida que las personas envejecen van perdiendo las capacidades necesarias para realizar las actividades de la vida diaria y son vulnerables a sufrir situaciones que atentan contra su salud.

Las caídas son un gran problema geriátrico que pueden conllevar a catastróficos resultados en quienes las sufren, se habla que un tercio de las personas mayores de 65 años que viven en la comunidad caen cada año y que cerca de la mitad de ellos sufren múltiples caídas (Moreland et al., 2003; Tinetti, 2003; Reyes-Ortiz et al., 2005). Existen diversas herramientas que valoran el riesgo de sufrir caídas como por ejemplo las pruebas funcionales de Tinetti y Timed up and go, ambos muy usados en centros geriátricos (Tinetti; Shumway-Cook et al., 2000). La transferencia desde la posición sedente a bípedo (TSB) o "Sit to stand" (en lenguaje anglosajón), es una tarea motora que requiere una adecuada capacidad motora y que determina el nivel de funcionalidad de una persona (Janssen et al., 2002). Esta tarea motora, ha sido muy usada en diversos estudios biomecánicos, en donde a partir de distintas variables cinéticas y cinemáticos es posible describir los parámetros biomecánicos involucrados en su ejecución (Burdett et al., 1985; Hughes \& Schenkman,1996; Hughes et al., 1996;

* Centro de Estudios del Movimiento Humano. Escuela de Kinesiología. Facultad de Medicina. Universidad Mayor. Santiago, Chile.

** Laboratorio de Biomecánica y Control Motor. Escuela de Kinesiología. Universidad Santo Tomás. Santiago. Chile.

**** Unidad de Caídas y Trastornos de la Marcha, Instituto Nacional de Geriatría, Santiago, Chile. 
GUZMÁN, R. A.; SALAZAR, H. A.; CEA, A.; MELIÁN, H. P.; CORDIER, B. \& SILVESTRE, R. A. Correlación entre el puntaje obtenido en la prueba "Timed up and go" y momentos articulares del miembro inferior registrados durante la transferencia de sedente a bípedo en adultos mayores con antecedentes de caídas frecuentes. Int. J. Morphol., 29(2):521-525, 2011.

Cahill \& Carr, 1999; Bernardi et at., 2004; Guzmán et al., 2009).

En el ambiente clínico el riesgo de caída es valorado mediante pruebas funcionales que muchas veces carecen de poder analítico, en pro de mejorar dichas herramientas es necesario describir cual de las pruebas disponibles puede ser sensible al comportamiento de parámetros biomecánicos relacionados con el riesgo de caídas, con la finalidad de conferirle a estas pruebas funcionales mayor poder analítico que ayuden a la mejor identificación de factores de riesgo, mejorando así el proceso de prevención de caídas. La prueba de "Timed up and go" es ampliamente utilizada en la prevención de caídas (Tinetti; Shumway-Cook et al.), la cual usa como variable de predicción el tiempo de ejecución de una tarea motora. El objetivo de este trabajo fue describir la correlación entre el puntaje obtenido en la prueba funcional "Timed up and go" y la capacidad de generar momentos articulares del miembro inferior obtenidos durante la ejecución de la transferencia de sedente a bípedo (TSB) en sujetos adultos mayores con antecedentes de caídas frecuentes.

\section{MATERIAL Y MÉTODO}

Mediante un muestreo no probabilístico y por conveniencia se obtuvo una muestra de treinta adultos mayores ( $\geq 65$ años) voluntarios ( 25 mujeres y 5 hombres), todos ingresados a la Unidad de Caídas y Trastornos de la Marcha del Instituto Nacional de Geriatría (ING). Los criterios de inclusión fueron: i) edad igual o mayor a 65 años, ii) historia de caídas frecuentes (dos o más caídas en los últimos seis meses (Gómez et al., 2009)) y iii) ser capaz de transferirse de sedente a bipedestación en forma autónoma y sin ayuda de los miembros superiores. Adicionalmente se aplicaron los siguientes criterios de exclusión: i) demencias de cualquier tipo, ii) enfermedades neurológicas centrales o periféricas, iii) antecedentes de patologías del aparato locomotor que involucren compromisos de la función o deformaciones de los miembros inferiores y/o del tronco (dolor lumbar crónico, artritis reumatoide no tratada, procesos artrósicos invalidantes, portador de prótesis totales o parciales; etc.), iv) alteraciones vestibulares, v) patologías visuales no corregidas, vi) obesidad o vii) desnutrición.

Todos los adultos mayores seleccionados fueron examinados en forma física y cognitiva por un médico y un terapeuta físico especialistas en el área de geriatría en dependencias del ING, a partir de dichos exámenes no se descartó a ninguno de los sujetos reclutados. Las caracte- rísticas de edad, talla y masa de la muestra son presentados en la Tabla I.

Tabla I. Promedio y desviación estándar (entre paréntesis) de la edad, talla y masa de treinta sujetos $(n=30)$ con antecedentes de caídas frecuentes.

\begin{tabular}{ccc}
\hline Edad (años) & Talla $(\mathbf{m})$ & Masa $(\mathbf{k g})$ \\
\hline $77.38(5.8)$ & $1.54(0.07)$ & $66.52(10.9)$ \\
\hline
\end{tabular}

Todos los sujetos participaron en forma voluntaria y se les solicitó su consentimiento por escrito una vez que fueron informados de los objetivos del trabajo, los procedimientos y los posibles riesgos de participar en el estudio. Los procedimientos llevados a cabo en este estudio fueron aprobados por el comité de ética del Instituto Nacional de Geriatría.

Los sujetos fueron evaluados con la prueba funcional de "timed up and go" mediante el siguiente procedimiento (Rockwood et al., 2000): se pidió a cada sujeto que tomara asiento en forma cómoda en una silla estándar sin apoya brazos de $42 \mathrm{~cm}$ de altura, frente a una indicación verbal por parte del evaluador se le solicitó al sujeto que se levantara desde la silla y caminar en línea recta hasta una distancia de 3 metros demarcados en el suelo, una vez que el sujeto llegó a este punto debió girar y regresar a sentarse en la silla, todo realizado de la manera más rápida posible. La prueba fue ejecutada tres veces separas de un minuto, el puntaje consignado fue el promedio del tiempo de las tres repeticiones. Todas las pruebas funcionales fueron controladas por un terapeuta físico especialista en el área de geriatría.

Los momentos articulares de tobillo, rodilla y cadera durante TSB fueron obtenidos a partir de datos cinemáticos y cinéticos registrados mediante un sistema de análisis de movimiento (APAS System. Ariel Dynamics, Trabuco Canyon, California. USA) dotado de dos plataformas de fuerza (AMS, Artificio. Santiago. Chile). Se utilizaron marcadores reflectantes pasivos fijados con cinta adhesiva doble faz hipoalergénica, para delimitar los segmentos del tronco, muslo, pierna y pie del hemicuerpo derecho, lo que permitió obtener la posición de dichos marcadores para el calculo las variables cinemáticas, momentos de inercia de los segmentos corporales (Dempster, 1955) y los momentos articulares según lo descrito por Winter (2005), el modelo mecánico utilizado es esquematizado en la Figura 1.

La evaluación biomecánica de la TSB se realizó con los brazos cruzados al pecho y sobre una silla de altura regulable a la interlinea articular de rodilla de cada sujeto, con la finalidad de homologar el nivel de exigencia de la TSB para cada individuo (Janssen et al.). La TSB fue realizada 


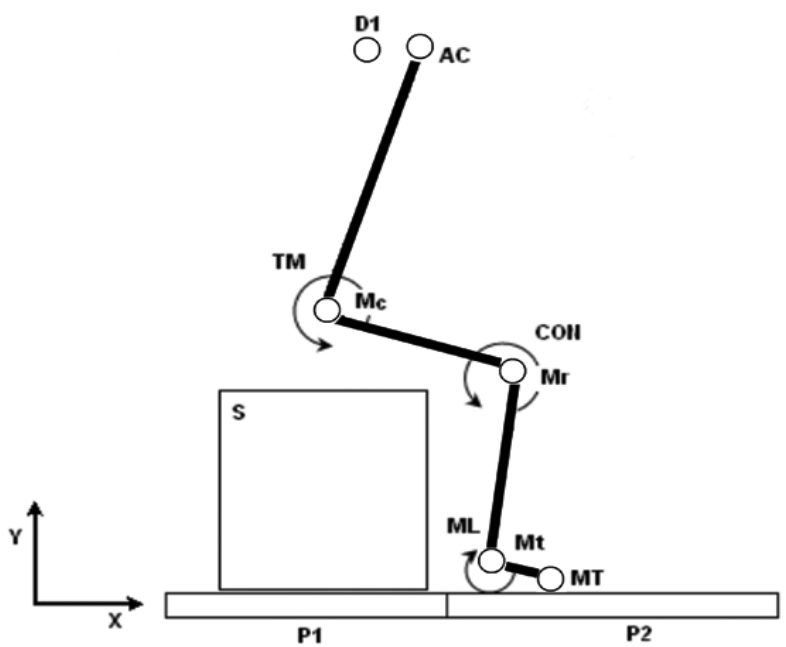

Fig. 1. Esquema del modelo mecánico utilizado para determinar los momentos articulares de tobillo, rodilla y cadera. Ubicación de los marcadores: D1: Proceso espinoso primera vértebra dorsal; AC: Borde lateral del acromion. TM: Trocánter mayor. CON: Tubérculo lateral del cóndilo lateral de fémur. ML: Maléolo fibular. MT: cabeza del segundo metatarsiano. P1 y P2: Plataforma de fuerza. $\mathrm{S}$ : Silla de altura regulable. M1: Torque neto en tobillo. M2: torque neto en rodilla. M3: torque neto en cadera.

en cuatro oportunidades, en las últimas tres se registraron las variables cinéticas y cinemáticas, a partir de las cuales se calcularon los momentos articulares normalizados al peso de cada sujeto. Los valores de momento articular para tobillo, rodilla y cadera fueron obtenidos a partir del promedio de los valores máximos para cada articulación en las tres pruebas ejecutadas.

Se utilizó el test de normalidad Shapiro-Wilk para establecer el supuesto de normalidad de los puntajes en la prueba "Timed up and go" y los momentos articulares de miembros inferiores, el nivel de confianza utilizado fue de
Tabla II. Media y desviaciones estándar (entre paréntesis) de los máximos momentos articulares de tobillo (Mt), rodilla(Mr), cadera $(\mathrm{Mc})$ y puntaje de la prueba funcional "Timed up and go" (TUG) en treinta sujetos $(n=30)$ con antecedentes de caídas frecuentes.

\begin{tabular}{cccc}
\hline TUG(seg) & Mt(Nm/kg) & Mr(Nm/kg) & Mc(Nm/kg) \\
\hline $13,46(4,24)$ & $0,62(0.24)$ & $0,86(0,36)$ & $1,55(0,38)$ \\
\hline
\end{tabular}

Tabla III. Coeficientes de correlación entre el tiempo registrado en la prueba de "Timed up and go" (TUG) y los máximos momentos articulares de tobillo (Mt), rodilla(Mr), cadera (Mc) obtenidos durante la transferencia de sedente a bípedo en adultos mayores con antecedentes de caídas frecuentes $(n=30)$. Entre paréntesis se expone los valores de $\mathrm{p}$. *Significancia estadística.

\begin{tabular}{cccc}
\hline & $\mathbf{M t}(\mathbf{N m} / \mathbf{k g})$ & $\mathbf{M r}(\mathbf{N m} / \mathbf{k g})$ & $\mathbf{M c}(\mathbf{N m} / \mathbf{k g})$ \\
\hline TUG[seg] & $-0,001(0.99)$ & $-0,39(0,03)^{*}$ & $-0,15(0.45)$ \\
\hline
\end{tabular}

$95 \%(\partial=0,05)$. La correlación entre los puntajes de la prueba funcional y los momentos articulares fue obtenida mediante las pruebas de Pearson y Spearman, para datos paramétricos y no paramétricos respectivamente, según el tipo de distribución de las variables a correlacionar.

\section{RESULTADOS}

El promedio y desviación estándar de los puntajes obtenidos en la prueba "Timed up and go" y los valores de momentos articulares son presentados en la Tabla II. Los coeficientes de correlación entre los puntajes de la prueba funcional y los momentos articulares son mostrados en la Tabla III.
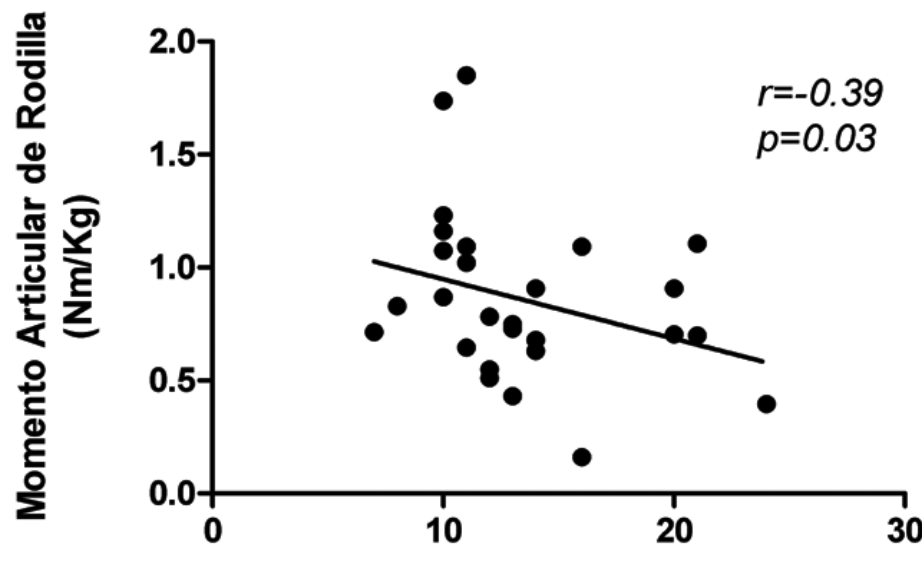

Fig. 2. Correlación entre el tiempo registrado en la prueba de "Timed up and go" (TUG) y el momento articular máximo de rodilla. Existió correlación significativa entre el tiempo de ejecución del la prueba "timed up and go" y el valor máximo del momento articular de rodilla $(\mathrm{r}=-0,39$, $\mathrm{p}=0,03$ ).

Tiempo de TUG (s) 
GUZMÁN, R. A.; SALAZAR, H. A.; CEA, A.; MELIÁN, H. P.; CORDIER, B. \& SILVESTRE, R. A. Correlación entre el puntaje obtenido en la prueba "Timed up and go" y momentos articulares del miembro inferior registrados durante la transferencia de sedente a bípedo en adultos mayores con antecedentes de caídas frecuentes. Int. J. Morphol., 29(2):521-525, 2011.

\section{DISCUSIÓN}

La alta prevalencia e incidencia de las caídas en las personas mayores de 60 años, sumadas al alto impacto socio-económico que conllevan estas hace necesario potenciar los procesos de prevención de caídas. Una de las piezas claves en dicho proceso es la valoración de su riesgo, para identificar a los sujetos más propensos y sobre ellos aplicar un plan de intervención. En la actualidad el riesgo de sufrir caídas en forma frecuentes es valorado habitualmente mediante un gran espectro de pruebas funcionales, de las cuales no todas han sido evaluadas en su sensibilidad y/o especificidad (Scott et al., 2007). Por otro lado muchas de las pruebas funcionales incluyen elementos de evaluación basados en la experiencia empírica del evaluador, agregando a dichas pruebas importantes componentes subjetivos en la valoración del riesgo de caídas. También muchas veces las pruebas funcionales carecen de poder analítico, por ejemplo, son incapaces de determinar que grupo muscular es el más afectado, en el caso que la debilidad muscular sea el factor de riesgo predominante. Sin embargo, una de las grandes cualidades de las pruebas funcionales es su bajo costo de implementación y facilidad de aplicación, característica que le otorgan la capacidad de ser aplicadas a grandes poblaciones de adultos mayores. La valoración de parámetros biomecánicos como los momentos articulares implica evaluar el desempeño del tejido muscular y de los mecanismos de control motor (Winter) en condiciones funcionales, a diferencia de las formas tradicionales de medición del desempeño muscular (ej. medición de la fuerza isométrica máxima), las que son valoradas en condiciones especiales muy distantes a la realidad de la ejecución de una tarea motora funcional como la marcha y la TSB. Sin embargo, la medición de parámetros biomecánicos está restringida por el alto costo de la instrumentación requerida y por la complejidad implicada en los proceso de valoración de dichos parámetros.

Sin duda que es necesario en el proceso de prevención de caídas contar con herramientas para valorar dicho riesgo y que complementariamente generen directrices para el diseño de medidas de intervención sobre los sujetos en riesgo. Por esta razón la motivación de este trabajo estuvo en contribuir a otorgar mayor poder analítico a una prueba funcional. Los resultados demostraron que el desempeño en la prueba de "timed up and go" se correlacionó con la capacidad de generar momento articular de rodilla $(r=-0,39$, $\mathrm{p}=0,03$ ) y no con dicha capacidad en el tobillo y la cadera. De esta forma los sujetos que registraron menos capacidad de generar momento articular de rodilla tardaron más en realizar la prueba de TUG, este hallazgo puede ser atribuido al rol de los músculos flexo-extensores de rodilla en la ejecución de la TSB y la marcha. Desde el punto de vista neurofisiológico la capacidad de generar momento articular depende de la capacidad de producir fuerza muscular, la que a su vez es dependiente de la cantidad de unidad motoras reclutadas y la frecuencia de descarga de estas (Winter). Por otro lado, desde el punto de vista geométrico la fuerza requerida para generar momento articular esta relacionada con el área de sección transversal (Winter). Nuestros resultados pueden indicar que el tiempo del TUG depende de la capacidad de generar momento en la articulación de rodilla. El proceso de sarcopenia y la perdida de unidades motoras rápidas (Macintosh et al., 2006), limitan la capacidad de generar momento articular, posiblemente dicha limitación podría tener mayor ponderación en sujetos con antecedentes de caídas frecuentes. Los resultados presentados en este trabajo podrían conferirle mayor poder analítico a la prueba de TUG, indicando que los sujetos con mayor tiempo de ejecución tendrían menor capacidad de generar momento en la articulación de rodilla, por lo cual su riesgo de caídas podría relacionarse entre otros factores con debilidad de los músculos flexo-extensores de rodilla. El momento articular de rodilla registrado durante la TSB, no solo depende de los músculos de la parte anterior del muslo (cuadriceps y vastos), sino también de los músculos del la parte posterior (isquiotibiales) los cuales al ser la TSB un tarea motora de cadena cinética cerrada invierten su rol flexor de rodilla a extensor de esta. Por lo cual el diseño de una posible intervención debería considerar ambos grupos musculares.

La principal conclusión de este trabajo fue que para los sujetos de la muestra evaluada, el tiempo de ejecución de la prueba "Timed up and go" fue indicador de la capacidad de generar momento articular por parte de los músculos flexo-extensores de rodilla en sujetos con antecedentes de caídas frecuentes.

GUZMÁN, R. A.; SALAZAR, H. A.; CEA, A.; MELIÁN, H. P.; CORDIER, B. \& SILVESTRE, R. A. Correlation between the scores of Timed up and go test and joints moments registered of lower limb during the sit-to-stand transfer in elderly with antecedents of frequent falls.Int. J. Morphol., 29(2):521-525, 2011.

SUMMARY: The changes that take place during aging predispose the elder adult to frequent falls. In clinical practice fall risk is assessed by clinical tests that many times lack analytical power, therefore making it necessary to describe which of the clinical tests are related to the analytical biomechanical parameters in order to assign such characteristics to these functional tests. The objective of this work was to describe the existence of a correlation between the score of the functional test "Timed up and go" (TUG) and the joint moments of lower limb obtained during the execution of the sit to stand (STS) transfer in elderly subjects with a history of frequent falls. A sample of 30 volunteers was obtained; all were elders with a history of frequent falls. They were assessed with the 
GUZMÁN, R. A.; SALAZAR, H. A.; CEA, A.; MELIÁN, H. P.; CORDIER, B. \& SILVESTRE, R. A. Correlación entre el puntaje obtenido en la prueba "Timed up and go" y momentos articulares del miembro inferior registrados durante la transferencia de sedente a bípedo en adultos mayores con antecedentes de caídas frecuentes. Int. J. Morphol., 29(2):521-525, 2011.

functional test of TUG. Also assessed were the joint moments of the lower limb with motion analysis system. There is significant correlation $(\mathrm{r}=0.39 ; \mathrm{p}=0.03)$ between the score obtained in the TUG and the maximum joint moment of the knee. For the sample assessed, the time of execution of the test "Timed up and go" was indicative of the capacity to generate a joint moment by the flexionextensor muscles of the knee in subjects with a history of frequent falls.

KEY WORDS: Fall; Elderly; Moment; Biomechanics.

\section{REFERENCIAS BIBLIOGRÁFICAS}

Bernardi, M.; Rosponi, A.; Castellano, V.; Rodio, A.; Traballesi, M.; Delussu, A. S. \& Marchetti, M. Determinants of sitto-stand capability in the motor impaired elderly. Electromyogr. Kinesiol., 14(3):401-10, 2004

Burdett, R. G.; Burdett, R. G.; Habasevich, R.; Pisciotta, J. \& Simon, S. R. Biomechanical comparison of rising from two types of chairs. Phys. Ther., 65(8):1177-83,1985.

Cahill, B. M. \& Carr, J. H. Inter-segmental coordination in sit-to-stand: an age cross-sectional study. Phys. Res. Int., 4(1):12-27,1999.

Dempster, W.T. "SDECE Requirements of the Seated Operator," WADC-TR-55-159, Wright Patterson Air Force Bass, 1955.

Gómez, P.; González, E.; Lázaro, M.; Lis, M. \& Ribera, J. M. Rev. Esp. Geriatr. Gerontol., 44:88-9, 2009.

Guzmán, R. A.; Prado, H. E.; Porcel Melián, H. \& Cordier, B. Diferencias en el desarrollo de momentum al levantarse desde una silla en adultos mayores con y sin historia de caídas frecuentes. Rev. Esp. Geriatr. Gerontol., 44(4):200-4, 2009.

Hughes, M. A.; Myers, B. S. \& Schenkman, M. L. The role of strength in rising from a chair in the functionally impaired elderly. J. Biomech., 29(12):1509-13, 1996.

Hughes, M. A. \& Schenkman, M. L. Chair rise strategy in the functionally impaired elderly. J. Rehabil. Res. Dev., 33(4):409-12, 1996.

Instituto Nacional de Estadística (INE). Enfoque estadistico, Adulto Mayor en Chile - Boletín informativo del Instituto Nacional de Estadísticas, 2007. Disponible en: http://www.ine.cl/canales/menu/boletines/ enfoques/ 2007/pdf/ine_adulto_mayor.pdf
Janssen, W. G.; Bussmann, H. B. \& Stam, H. J. Determinants of the sit-to-stand movement: A review. Phys. Ther., 82(9):866-79,2002.

Maclntosh, B. R.; Gardiner, P. F. \& McComas, A. J. Skeletal Muscle from and function. $2^{\text {nd }} \mathrm{ed}$. Champaign, Human Kinetics, 2006. pp.322-9.

Moreland, J.; Richardson, J.; Chan, D. H.; O'Neill, J.; Bellissimo, A.; Grum, R. M. \& Shanks, L. EvidenceBased Guidelines for the Secondary Prevention of Falls in Older Adults. Gerontology, 49(2):93-116,2003.

Reyes-Ortiz, C. A.; Al Snih, S. \& Markides, K. S. Falls among elderly persons in Latin América and the Caribbean and among elderly Mexican-Americans. Rev. Panam. Salud Publica, 17(5-6):362-9, 2005.

Rockwood, K.; Awalt, E.; Carver, D. \& Macknightm C. Feasibility and measurement properties of the functional reach and the timed up and go tests in the Canadian Study of Health and Aging. J. Gerontol. A. Biol. Sc.i Med. Sci.; 55(2):M70-3, 2000.

Scott, V.; Votova, K.; Scanlan, A. \& Close, J. Multifactorial and functional mobility assessment tool for fall risk among older adults in community, home-support, longterm and acute care settings. Age Ageing, 36(2):130-9, 2007.

Shumway-Cook, A.; Brauer, S. \& Woollacott, M. Predicting the probability for falls in community-dwelling older adults using the Timed up and go test. Phys. Ther., 80(9):896-903, 2000.

Tinetti, M. E. Clinical Practice: Preventing Falls in Elderly Persons. N. Engl. J. Med., 348(1):42-9, 2003.

Winter, D. A. Biomechanics and Motor Control of human Movement. 3TH edn. New Yersey, Wiley and Sons, 2005. pp.106-10.

\section{Dirección para correspondencia: \\ Rodrigo Guzmán Venegas \\ Centro de Estudios del Movimiento Humano \\ Universidad Mayor \\ Escuela de Kinesiología}

Facultad de Medicina

Camino La Pirámide 5750 Recibido : 23-07-2010

Huechuraba, Santiago

Aceptado: 11-02-2011

CHILE

Email: rodrigoguzmanvenegas@gmail.com 